\title{
Long-term low-dose erythromycin in patients with unexplained chronic cough: a double-blind placebo controlled trial
}

\author{
Nadia Yousaf, ${ }^{1}$ William Monteiro, ${ }^{1}$ Debbie Parker, ${ }^{1}$ Sergio Matos, ${ }^{2}$ Surinder Birring, ${ }^{3}$ \\ lan D Pavord ${ }^{1}$
}

1 Institute for Lung Health, Glenfield Hospital, University Hospitals of Leicester NHS Trust, Leicester, UK ${ }^{2}$ Institute of Electronics and Telematics Engineering (IEETA), University of Aveiro, Aveiro, Portugal

${ }^{3}$ Kings College Hospital, Denmark Hill, London, UK

\section{Correspondence to}

Professor lan D Pavord, Institute for Lung Health, Glenfield Hospital, University Hospitals of Leicester NHS Trust, Groby Road, Leicester LE3 90P, UK; ian.pavord@uhl-tr.nhs.uk

Received 14 May 2010 Accepted 5 August 2010 Published Online First 21 October 2010
Aims Unexplained chronic cough is a common condition with no satisfactory treatments. Previous work has suggested that cough may be linked to neutrophilic airway inflammation. This study tested the hypothesis that long-term low-dose erythromycin reduces the induced sputum neutrophil count and $24 \mathrm{~h}$ cough frequency in patients with unexplained chronic cough. Methods 30 patients with an unexplained chronic cough lasting more than 8 weeks were randomly assigned to take $250 \mathrm{mg}$ erythromycin once daily $(n=15)$ or placebo $(n=15)$ for 12 weeks in a double-blind parallel group study. Cough frequency, cough reflex sensitivity and cough severity were assessed at baseline, 6, 12 and 24 weeks. The primary outcome measure was change in $24 \mathrm{~h}$ cough frequency at 12 weeks.

Results There was no difference in the change in cough frequency between the erythromycin and placebo groups at 12 weeks (mean difference in fold change 1.1; 95\% Cl 0.7 to $1.5 ; p=0.585$ ) or at other times. There was a statistically significant between-treatment difference in the change in sputum neutrophils at 12 weeks $(-10.2 \%$ vs $+6.6 \%$ with erythromycin and placebo; mean difference $16.8 \%$; $95 \% \mathrm{Cl} 1.6$ to $32.1 ; p=0.03$ ) but not at other times. There was no difference in the change in other measures of cough between treatments.

Conclusions Treatment with low-dose erythromycin for 12 weeks reduces the induced sputum neutrophil count but not cough frequency or severity in patients with unexplained chronic cough.

No cause is found for chronic cough in up to $40 \%$ of patients presenting to specialist cough centres. ${ }^{1-3}$ Patients with unexplained chronic cough remain a challenge to manage as treatment options are limited. Patients are mainly women and they have a higher than expected prevalence of organ-specific autoimmune disease; many report the onset of cough around the menopause. ${ }^{3-5}$ Quality of life studies have shown that in some domains of a generic quality of life score, patients with unexplained chronic cough have impairment equivalent to that seen in patients with severe chronic obstructive pulmonary disease. ${ }^{6}$

Cross-sectional studies have shown that patients with unexplained chronic cough cough on average 477 times/24 h, 13 times more frequently than controls. ${ }^{7}$ They have an increased cough reflex to inhaled capsaicin, an induced sputum neutrophilia, and raised concentration of mediators associated with neutrophilic airway inflammation, including interleukin 8 (IL-8), tumour necrosis factor alpha and prostaglandin $\mathrm{E}_{2}\left(\mathrm{PGE}_{2}\right) .^{8-10}$ We have previously noted a significant, independent association between the induced sputum neutrophil count and $24 \mathrm{~h}$ cough frequency ${ }^{11}$ suggesting that there may be a causal link between neutrophilic airway inflammation and cough.

Long-term low-dose macrolides have been used successfully to treat respiratory conditions associated with neutrophilic inflammation of the airways, and have been shown to reduce the induced sputum neutrophil count. ${ }^{12-14}$ We conducted a randomised double-blind placebo control study to test the hypothesis that $250 \mathrm{mg}$ erythromycin given for 3 months reduces neutrophilic airway inflammation and $24 \mathrm{~h}$ cough frequency in patients with unexplained chronic cough.

\section{METHODS \\ Subjects}

Patients with a chronic cough lasting more than 8 weeks were recruited from consecutive consenting patients attending the Leicester cough clinic from May to November 2008. They had normal spirometry, a provocative concentration of methocholine required to cause a $20 \%$ fall in forced expiratory volume in $1 \mathrm{~s}$ of more than $8 \mathrm{mg} / \mathrm{ml}$, a normal induced sputum eosinophil count and a normal high resolution CT scan of the thorax. All subjects had failed treatment trials with a proton pump inhibitor with or without alginate and a nasal steroid spray with or without antihistamine of 2 months or more as per British Thoracic Society guidelines. $^{15}$

Exclusion criteria were current smokers or past smokers with a greater than 10 pack-year history, those with a history of intolerance to macrolide antibiotics and pregnant/breastfeeding women. No patients took any specific therapy for cough during the study. Written consent was obtained from all patients and the study was approved by the Leicestershire, Northamptonshire and Rutland research ethics committee and the Medicines and Healthcare Regulatory Authority. The international standard randomised control trial number for this study is ISRCTN75393495.

\section{Study design}

Patients attended for two initial baseline visits. Spirometry was performed on the first visit. The Leicester cough monitor was then attached and the patients were asked to return $24 \mathrm{~h}$ later. On the 
second visit the Leicester cough monitor was removed after which the patients underwent a capsaicin cough challenge, sputum induction and completed the Leicester cough questionnaire (LCQ) and a cough visual analogue score (VAS). They were then randomly assigned to receive $250 \mathrm{mg}$ erythromycin stearate or placebo (cellulose) as identical capsules once a day for 12 weeks in a double-blind parallel group study. Baseline measurements were repeated at the same time of day, $2 \mathrm{~h}$ after ingestion of the study drug at 6,12 and 24 weeks.

Randomisation was carried out before commencement of the study by pharmacy independent of blinded trial staff. Randomisation was in blocks of four (two erythromycin/two placebo) and numbers from 1 to 6 from a Geigy random number generating table. Patients were automatically dispensed the next allocated treatment.

\section{Analysis}

Cough frequency, concentration of capsaicin required to cause two (C2) and five (C5) coughs, sputum eosinophil differential count and sputum supernatant IL-8 concentrations were log transformed to assume normality before analysis. Our primary endpoint was a change in log $24 \mathrm{~h}$ cough frequency from baseline to 12 weeks as we anticipated that any drug effect would be greatest at this time. Secondary endpoints were changes in induced sputum neutrophils differential cell count, sputum bacterial colonies, $\log$ C2, C5, cough VAS and LCQ. Mixed design analysis of covariance with sidicks contrast ${ }^{16}$ was used to compare results with the pre-treatment result used as the covariate to account for baseline differences. We performed an intention to treat analysis; subjects withdrawing after randomisation were assigned post-treatment values using the last value carried forward. Our original target recruitment population was 20 patients per treatment arm. This gave us $80 \%$ power to detect a $50 \%$ reduction in $24 \mathrm{~h}$ cough frequency $(\alpha=0.05)$ with active treatment compared with placebo based on our previous data showing a mean log $24 \mathrm{~h}$ cough frequency of 2.68 and a within-subject log SD of 0.3 . Statistical analysis was performed using SPSS 16.

\section{RESULTS}

Thirty-five consecutive patients diagnosed with unexplained chronic cough attending the Leicester cough clinic were approached between May and November 2008. Thereafter recruitment had to be closed because the supplier of erythromycin and placebo ceased production of matched placebo. Thirty consented to take part in the study and were randomly assigned (erythromycin $n=15$, placebo $n=15$ ). Two patients withdrew from the study due to personal reasons. Twenty-eight patients completed the study (erythromycin $n=13$, placebo $\mathrm{n}=15$; figure 1).

Patients' baseline demographic information is shown in table 1. Coughs per $24 \mathrm{~h}$ were statistically significantly higher at baseline in the placebo group compared with the erythromycin group. All other baseline characteristics were comparable in both groups. Induced sputum samples were obtained in 76 out of 90 samples $(84 \%)$. All patients who completed the study showed greater than $95 \%$ compliance with the treatment prescribed. Two patients in the placebo group reported abdominal discomfort at the 6 week visit, which resolved by the 3 month visit. One patient in the erythromycin group reported dizziness at the second visit, which resolved within a week.

Results are shown in table 2. Geometric mean $24 \mathrm{~h}$ cough frequency at baseline and 12 weeks was 536 and 390 (mean fold difference $0.7 ; 95 \%$ CI 0.6 to 0.9 ) with placebo and 353 and 243

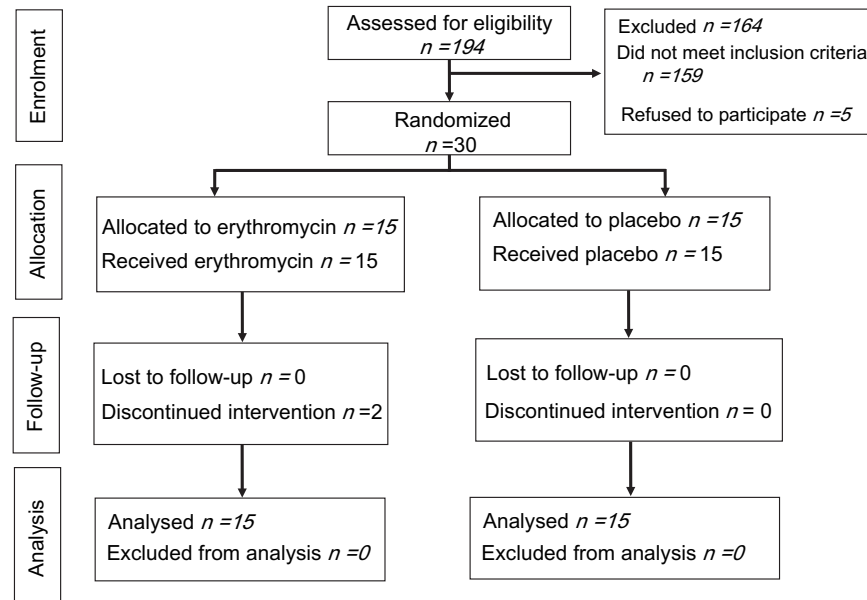

Figure 1 Patient flow with erythromycin and placebo treatment.

(mean fold difference $0.7 ; 95 \%$ CI 0.5 to 1.0 ) with erythromycin. After adjusting for baseline differences there was no difference in the change in cough frequency between placebo and erythromycin groups at 12 weeks (mean difference in fold change 1.1; $95 \%$ CI 0.7 to $1.5 ; p=0.585$ ) or at other times (figure 1 ). The induced sputum neutrophil count reduced from $67.8 \%$ to $56.6 \%$ after 12 weeks treatment with erythromycin. There was a statistically significant between-treatment difference in the change in sputum neutrophils at 12 weeks $(-10.2 \%$ vs $+6.6 \%$ with erythromycin and placebo, respectively; mean difference $16.8 \%$; $95 \%$ CI 1.6 to $32.1 \%$; $p=0.03$ ) but no difference at other times (table 2, figure 2). There was no difference in the change in LCQ, cough VAS, log C2 or log C5 between treatments (figure 2). Haemophilus influenzae, Streptococcus pneumoniae and Moraxella catarrhalis were not isolated from sputum samples at any time point, and the log bacterial colony forming units was unchanged with active and placebo treatment (table 2). Sufficient paired sputum supernatant was available to measure IL-8 in six patients randomly assigned to erythromycin and 10 to placebo. The geometric mean sputum supernatant IL-8 concentration was 9.15 and $8.09 \mathrm{ng} / \mathrm{ml}$ before and 12 weeks

Table 1 Mean (SD) baseline characteristics

\begin{tabular}{lll}
\hline & Erythromycin $(\mathbf{n}=15)$ & Placebo (n=15) \\
\hline No of women & 13 & 11 \\
Age, years & $63(9)$ & $61(9)$ \\
Duration of cough, years & $12(4.2)$ & $11.1(4.8)$ \\
Body mass index, kg/m & $27.5(4.6)$ & $26.1(4.9)$ \\
Percentage predicted FEV ${ }_{1}$ & $95(10)$ & $96(10)$ \\
FEV $/$ FVC & $0.77(0.07)$ & $0.76(0.04)$ \\
No of coughs in $24 \mathrm{~h}^{*}$ & $389(0.33)$ & $525(0.33)$ \\
Total cells/g of selected sputum & $3.3 \times 10^{6}\left(1.9 \times 10^{6}\right)$ & $3.1 \times 10^{6}\left(2.5 \times 10^{6}\right)$ \\
Percentage sputum neutrophils & $68(15)$ & $65(26)$ \\
Percentage sputum eosinophils* & $0.6(0.4)$ & $0.54(0.37)$ \\
Sputum IL-8 (ng/ml)* & $9.15(0.27)$ & $12.11(0.58)$ \\
Bacterial CFU/ml* & $47(0.25)$ & $29(0.28)$ \\
C2 $(\mu \mathrm{mol} /)^{*}$ & $1.1(0.69)$ & $0.62(0.91)$ \\
C5 $(\mu \mathrm{mol} /)^{*}$ & $2.88(0.55)$ & $4.17(0.83)$ \\
LCO score & $11.65(1.97)$ & $11.86(2.95)$ \\
Cough VAS $(\mathrm{mm})$ & $57(18)$ & $52(17)$ \\
\hline
\end{tabular}

*Geometric mean (log SD).

CFU, bacterial colony forming units; $\mathrm{C} 2$ and $\mathrm{C} 5$, concentration of capsaicin required to cause two and five coughs; FEV 1 , forced expiratory volume in $1 \mathrm{~s}$; FVC, forced vital capacity; IL-8, interleukin 8; LCQ, Leicester cough questionnaire; VAS, cough visual analogue score. 
Table 2 Mean (SD) and * geometric mean (log SD) fold change in parameters from baseline to immediately post-treatment at 12 weeks with between-group differences and $95 \% \mathrm{Cl}$

\begin{tabular}{|c|c|c|c|}
\hline & \multicolumn{2}{|c|}{$\begin{array}{l}\text { Change from baseline } \\
\text { to } 3 \text { months }\end{array}$} & \multirow[b]{2}{*}{ Difference $(95 \% \mathrm{Cl})$} \\
\hline & Placebo & Erythromycin & \\
\hline${ }^{*}$ Coughs/24 h & $0.73(0.66)$ & $0.67(0.29)$ & $1.1(0.7$ to 1.5$)$ \\
\hline Sputum neutrophils (\%) & $+6.6(23)$ & $-10.2(33)$ & 16.8 (1.6 to 32.1$)$ \\
\hline${ }^{*} \mathrm{C} 2(\mu \mathrm{mol} / \mathrm{l})$ & $1.1(0.4)$ & $1.6(0.06)$ & $0.7(0.4$ to 1.3$)$ \\
\hline${ }^{*} \mathrm{C} 5(\mu \mathrm{mol} / \mathrm{l})$ & $1.2(0.13)$ & $0.9(0.25)$ & $1.3(0.9$ to 2.0$)$ \\
\hline VAS (mm) & +2 (29) & $-12(33)$ & $10(-11$ to 33$)$ \\
\hline LCO & $+1.8(3.8)$ & $+1.8(3.8)$ & $0(-2$ to 2$)$ \\
\hline${ }^{*} \mathrm{CFU} / \mathrm{ml}$ & $1.17(0.56)$ & $0.65(0.69)$ & 1.8 (0.6 to 5.8$)$ \\
\hline${ }^{*}$ Sputum IL-8 (ng/ml) & $2.14(0.69)$ & $0.75(0.23)$ & $2.9(0.8$ to 9.5$)$ \\
\hline
\end{tabular}

CFU, bacterial colony forming units; $\mathrm{C} 2$, concentration of capsaicin required to induce two coughs; C5, concentration of capsaicin required to induce five coughs; IL-8, interleukin 8; LCO, Leicester cough questionnaire; VAS, cough visual analogue score.

after erythromycin treatment and 12.11 and $26.10 \mathrm{ng} / \mathrm{ml}$ before and 12 weeks after placebo treatment (mean difference in fold change $2.9 ; 95 \%$ CI 0.9 to $9.5 ; \mathrm{p}=0.08$; table 2 ).

\section{DISCUSSION}

Unexplained chronic cough is a common condition responsible for considerable morbidity. ${ }^{1-3}$ There are currently no satisfactory treatments. Our study is one of the first to evaluate a potential new treatment. We used a wide range of objective and subjective measures of cough severity, as have been recommended. ${ }^{1}$ We found no difference between the placebo and erythromycin group in any measure of cough severity, although there was a reduction in sputum neutrophil percentage with erythromycin at the end of the treatment period. Although we were not able to reach our target recruitment population, our study was sufficiently powered to exclude a halving in $24 \mathrm{~h}$ cough frequency and a clinically important reduction in LCO, ${ }^{17}$ making it unlikely that we missed a clinically important effect. Our findings imply that cough in patients with unexplained chronic cough is caused by a mechanism independent of neutrophilic airway inflammation.

Our rationale for investigating the effect of erythromycin was based on evidence from cross-sectional data indicating that neutrophilic airway inflammation is present in patients with unexplained cough, ${ }^{10}$ and is independently associated with $24 \mathrm{~h}$ cough frequency in a mixed population. ${ }^{11}$ Moreover, mediators associated with neutrophilic airway inflammation, notably $\mathrm{PGE}_{2}$, have been implicated in causing a heightened cough reflex. ${ }^{18} 19$ Our finding that long-term macrolide therapy reduced the sputum neutrophil count is consistent with findings in other inflammatory airway diseases. ${ }^{12}{ }^{14}$ However, the anti-inflammatory effect of erythromycin was not associated with clinical benefit. Treatment reduced the sputum neutrophil count into the normal range, ${ }^{20}$ making it unlikely that the absence of clinical effect was due to insufficient dose, a suboptimum macrolide regime or insufficient suppression of inflammation, although these possibilities cannot be excluded with complete confidence. Further work evaluating a wider dose range, potentially more efficacious macrolide regimes and more efficacious inhibitors of neutrophilic airway inflammation will be required to do this.

The mechanism of the anti-inflammatory effect of long-term low-dose macrolide antibiotics in inflammatory airway diseases is incompletely understood. We did not detect clinically significant pathogens on sputum culture and bacterial colony forming units were low and unaffected by treatment, suggesting that the
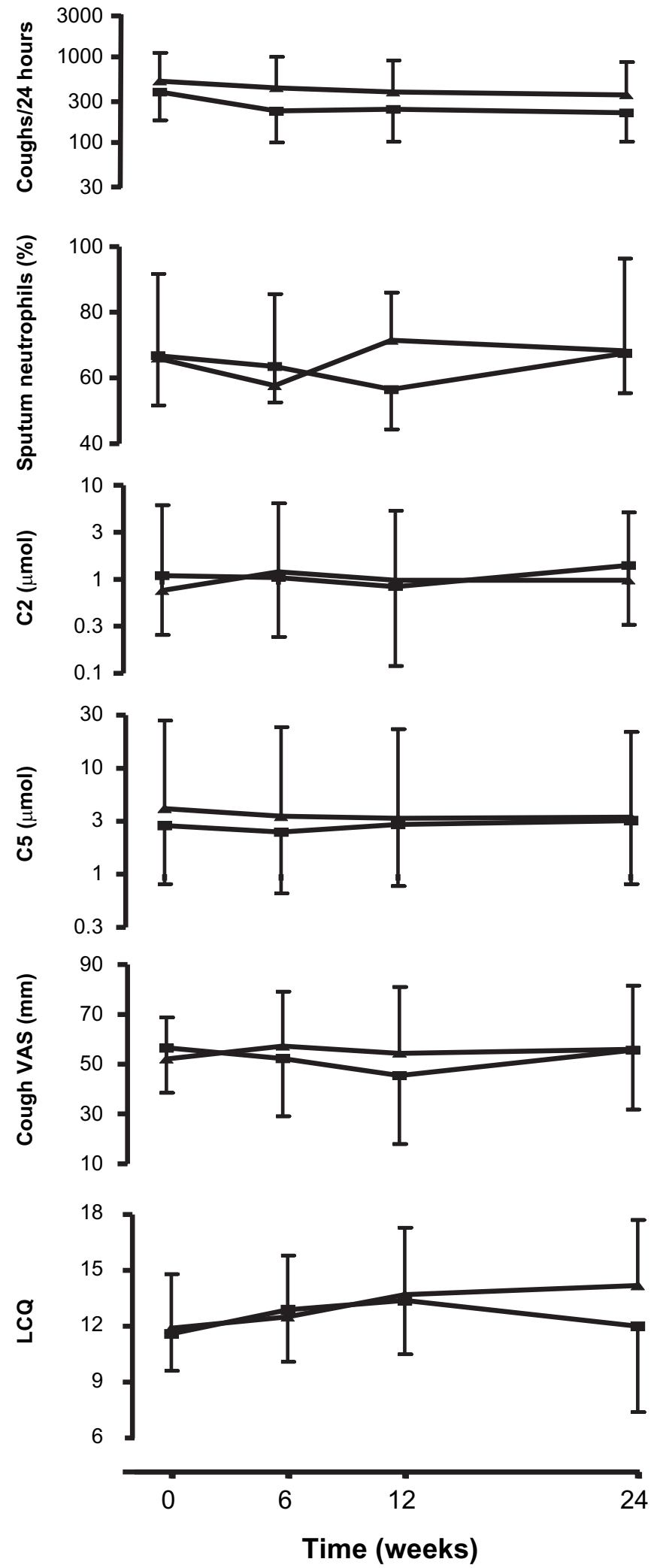

Figure 2 Geometric mean (log SEM) number of coughs in $24 \mathrm{~h}$, concentration of capsaicin required to cause two (C2) and five (C5) coughs and mean (SEM) induced sputum neutrophils \%, cough visual analogue score (VAS) and Leicester cough questionnaire (LCO) score. Triangles represent placebo treatment, squares erythromycin.

effect was not due to the effect of macrolide antibiotics on detectable pathogens. An inhibitory effect on intracellular organisms such as Mycoplasma pneumoniae or Chlamydia 
pneumoniae is possible. We found a trend to reduced IL-8 concentrations with erythromycin treatment compared with placebo, raising the possibility that inhibition of this cytokine might be important. Studies of human airway epithelium in vitro show that erythromycin inhibits the expression of IL-8, ${ }^{21}$ and there is evidence that macrolide antibiotics reduce sputum IL-8 concentrations in asthma ${ }^{12}$ supporting such an effect. The sputum supernatant data should be interpreted cautiously as only half of the patient population had sufficient paired sputum supernatant to do mediator assays and we cannot exclude the possibility that this represented a biased population. The lack of available sputum supernatant also means that we were unable to measure other mediators potentially more relevant to the genesis of cough such as $\mathrm{PGE}_{2}$. This means that we cannot exclude the possibility that erythromycin had a differential inhibitory effect on neutrophilic airway inflammation and associated tussive mediators.

Our study is notable in that it used an automated measurement of cough frequency as an outcome measure. We have previously shown that the Leicester cough monitor is a quick and valid method of measuring cough frequency. ${ }^{7}$ Detailed evaluation of day-time cough recordings has shown a sensitivity of $80-90 \%$ and a specificity of $99.8 \%$, and automated $24 \mathrm{~h}$ cough counts have been shown to correlate closely with blinded manual counts. ${ }^{7}$ An unexpected finding of the current study was the large reduction in $24 \mathrm{~h}$ cough frequency and improvement in LCQ seen with placebo treatment. Regression to the mean is an unlikely explanation for this reduction as our patients reported chronic cough for many years before study entry. The existence of such an effect supports suggestions that the traditional uncontrolled 'treatment trial' approach to the evaluation of patients with chronic cough is flawed, particularly if $24 \mathrm{~h}$ cough frequency is used to assess treatment response. ${ }^{1}$

The cause of unexplained chronic cough remains unclear and therefore there is uncertainty as to where drug treatment should be targeted. Opioid drugs have been used as an antitussive agent but results are mixed, ${ }^{22} 23$ and there is uncertainty about how acceptable more potent opiates would be to patients. Nebulised local anaesthetic agents ${ }^{24}$ and speech therapy intervention ${ }^{25}$ with an emphasis on voluntary cough suppression have been shown to be effective in preliminary studies, although those studies were unblinded and did not evaluate cough in sufficient detail to be sure about the benefits or the mechanism of the effect. There thus remains an important unmet need for effective treatments for an important group of patients. Our study should help researchers design and power future clinical trials; it should also direct researchers away from treatments targeting neutrophilic airway inflammation.

Acknowledgements The authors would like to thank the willing volunteers and the staff in the lung function department at Glenfield Hospital for help with the measurements and sputum induction.

Funding Institutional funds.

\section{Competing interests None}

\section{Patient consent Obtained.}

Ethics approval This study was conducted with the approval of the Leicestershire Northamptonshire and Rutland research ethics committee and the Medicines and Healthcare Regulatory Authority.

Provenance and peer review Not commissioned; externally peer reviewed.

\section{REFERENCES}

1. Pavord ID, Chung KF. Management of chronic cough. Lancet 2008;371:1375-84.

2. Haque RA, Usmani OS, Barnes PJ. Chronic idiopathic cough: a discrete clinical entity? Chest 2005;127:1710-13.

3. McGarvey LP. Idiopathic chronic cough: a real disease or a failure of diagnosis? Cough 2005;1:9.

4. Birring SS, Brightling CE, Symon FA, et al. Idiopathic chronic cough: association with organ specific autoimmune disease and bronchoalveolar lymphocytosis. Thorax 2003:58:1066-70.

5. Birring SS, Murphy AC, Scullion JE, et al. Idiopathic chronic cough and organspecific autoimmune diseases: a case-control study. Respir Med 2004;98:242-6.

6. French CL, Irwin RS, Curley FJ, et al. Impact of chronic cough on quality of life. Arch Intern Med 1998;158:1657-61.

7. Birring SS, Fleming T, Matos S, et al. The Leicester Cough Monitor: preliminary validation of an automated cough detection system in chronic cough. Eur Respir J 2008;31:1013-18.

8. Prudon B, Birring SS, Vara DD, et al. Cough and glottic-stop reflex sensitivity in health and disease. Chest 2005;127:550-7.

9. Morice $\mathbf{A H}$, Kastelik JA, Thompson R. Cough challenge in the assessment of cough reflex. Br J Clin Pharmacol 2001:52:365-75.

10. Jatakanon A, Lalloo UG, Lim S, et al. Increased neutrophils and cytokines, TNFalpha and IL-8, in induced sputum of non-asthmatic patients with chronic dry cough Thorax 1999;54:234-7.

11. Yousaf N, Matos S, Birring SS, et al. Factors affecting cough frequency in a mixed population. Thorax 2009;64:A151.

12. Simpson JL, Powell H, Boyle MJ, et al. Clarithromycin targets neutrophilic airway inflammation in refractory asthma. Am J Respir Crit Care Med 2008:177:148-55.

13. Seemungal TA, Wilkinson TM, Hurst JR, et al. Long-term erythromycin therapy is associated with decreased chronic obstructive pulmonary disease exacerbations. Am J Respir Crit Care Med 2008:178:1139-47.

14. Idris SF, Chilvers ER, Haworth $C$, et al. Azithromycin therapy for neutrophilic airways disease: myth or magic? Thorax 2009;64:186-9.

15. Morice AH, McGarvey L, Pavord I. Recommendations for the management of cough in adults. Thorax 2006;61(Suppl 1):i1-24.

16. Field A. Discovering statistics using SPSS. 2nd edn. Chapter 9. London: Sage, 2005

17. Raj AA, Pavord ID, Birring SS. Chronic cough IV: what is the minimal important difference for the Leicester Cough Questionnaire? Handb Exp Pharmacol 2009:287:311-20.

18. Birring SS, Parker D, Brightling CE, et al. Induced sputum inflammatory mediator concentrations in chronic cough. Am J Respir Crit Care Med 2004;169:15-19.

19. Stone R, Barnes PJ, Fuller RW. Contrasting effects of prostaglandins E2 and F2 alpha on sensitivity of the human cough reflex. J Appl Physiol 1992;73:649-53.

20. Thomas RA, Green RH, Brightling CE, et al. The influence of age on induced sputum differential cell counts in normal subjects. Chest 2004;126:1811-14.

21. Takizawa H, Desaki M, Ohtoshi T, et al. Erythromycin modulates IL-8 expression in normal and inflamed human bronchial epithelial cells. Am J Respir Crit Care Med 1997:156:266-71.

22. Morice AH, Menon MS, Mulrennan SA, et al. Opiate therapy in chronic cough. Am J Respir Crit Care Med 2007:175:312-15.

23. Smith J, Owen E, Earis $\mathrm{J}$, et al. Effect of codeine on objective measurement of cough in chronic obstructive pulmonary disease. J Allergy Clin Immunol 2006:117:831-5.

24. Howard $\mathbf{P}$, Cayton RM, Brennan SR, et al. Lignocaine aerosol and persistent cough Br J Dis Chest 1977:71:19-24.

25. Vertigan AE, Theodoros DG, Gibson PG, et al. Efficacy of speech pathology management for chronic cough: a randomised placebo controlled trial of treatment efficacy. Thorax 2006;61:1065-9. 